\title{
Experiments and Simulations of a Shocked Right-Cylinder Perturbation
}

\author{
L. M. Logory \\ K. T. Cheng \\ D. E. Smith
}

September 4, 1998

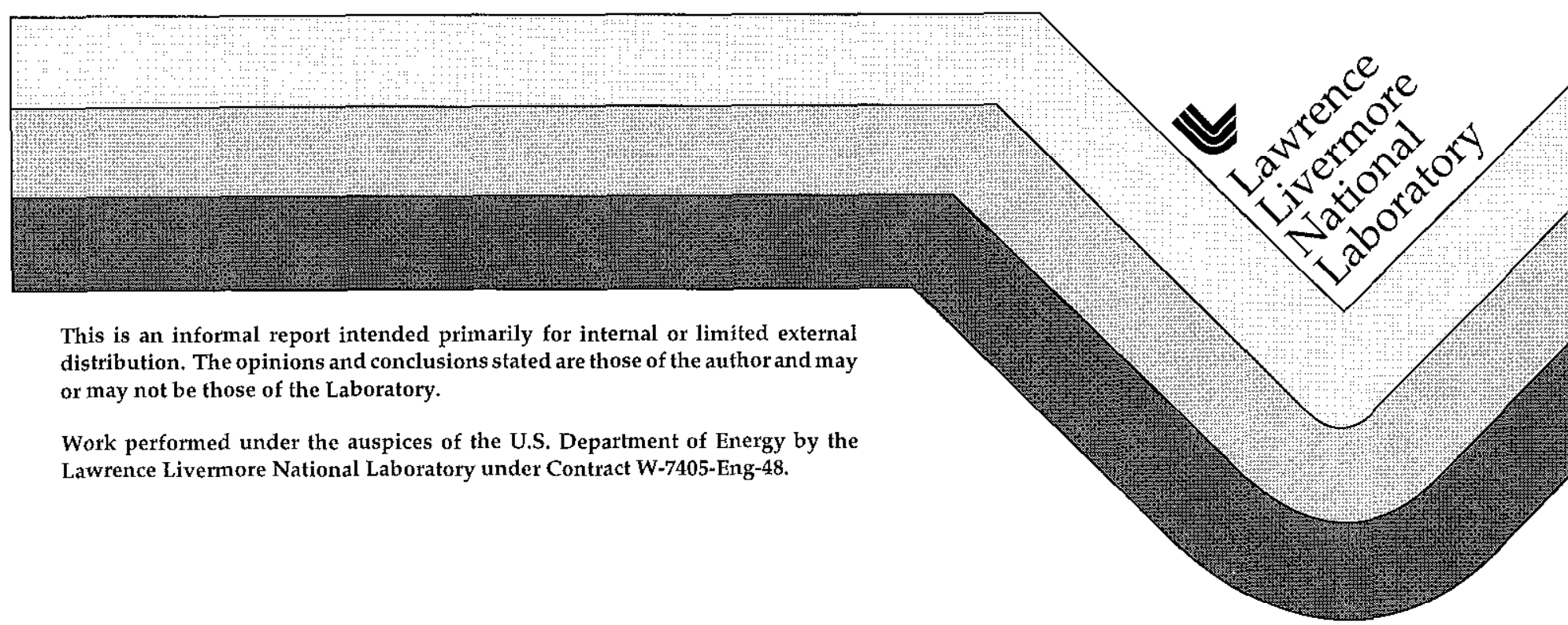




\section{DISCLAIMER}

This document was prepared as an account of work sponsored by an agency of the United States Government. Neither the United States Government nor the University of California nor any of their employees, makes any warranty, express or implied, or assumes any legal liability or responsibility for the accuracy. completeness, or usefulness of any information, apparatus, product, or process disclosed, or represents that its use would not infringe privately owned rights. Reference herein to any specific commercial product, process, or service by trade name, trademark, manufacturer, or otherwise, does not necessarily constitute or imply its endorsement, recommendation, or favoring by the United States Government or the University of California. The views and opinions of authors expressed herein do not necessarily state or reflect those of the United States Government or the University of California, and shall not be used for advertising or product endorsement purposes. 


\title{
Experiments and simulations of a shocked right-cylinder perturbation
}

\author{
L.M. Logory, K.T. Cheng, D.E. Smith \\ Lawrence Livermore National Laboratory, A-Division \\ Livermore, California 94551
}

We have conducted a series of experiments using the Nova laser facility at LLNL and corresponding simulations using the two-dimensional, Arbitrary Lagrangian-Eulerian hydrodynamics code CALE. The purpose of this work was to study the shock-driven, hydrodynamic behavior of a right-cylinder perturbation. The accuracy of our simulations is examined by comparison with the experiments.

\section{NOVA EXPERIMENT DESIGN}

A series of Nova experiments has been designed to investigate the hydrodynamic response of a rightcylinder perturbation to shock loading. The experimental configuration is shown in Fig. 1. Eight beams of the Nova laser are used to heat the interior of a stretched scale-1 hohlraum. The laser drive beams contain a total energy of $20 \mathrm{~kJ}$ in a $1 \mathrm{~ns}$ square pulse of $0.35 \mu \mathrm{m}$ wavelength light. The laser beams deposit their energy on the interior walls of the hohlraum and this energy is then re-emitted as $\mathrm{X}$ rays having a quasi-Planckian distribution with a peak temperature of approximately $230 \mathrm{eV}$.

An experimental package is attached to a flat region located on the mid-plane of the hohlraum. The package assembly consists of a $750 \mu \mathrm{m}$ inner-diameter, $950 \mu \mathrm{m}$ outer-diameter, $2000 \mu \mathrm{m}$ long beryllium tube which is affixed to the hohlraum with epoxy. The package is centered over a $750 \mu \mathrm{m}$ diameter hole in the flat which exposes the package to the interior of the hohlraum. Contained within the beryllium tube is a series of plastic and foam cylinders in a stacked design. Beginning at the hohlraum end, the package contains the following materials: a $200 \mu \mathrm{m}$ long, 2.0 atomic percent bromine-doped plastic ablator $\left(\rho=1.22 \mathrm{~g} / \mathrm{cm}^{3}\right)$, a $200 \mu \mathrm{m}$ long plastic material $\left(\rho=1.05 \mathrm{~g} / \mathrm{cm}^{3}\right)$, a $200 \mu \mathrm{m}$ diameter $\times 200 \mu \mathrm{m}$ long, 6.25 atomic percent bromine-doped plastic right-cylinder perturbation $\left(\rho=1.57 \mathrm{~g} / \mathrm{cm}^{3}\right)$, and a carbon foam payload $\left(\rho=0.1 \mathrm{~g} / \mathrm{cm}^{3}\right)$. The ablator material was doped with bromine in order to reduce radiative preheat to the bulk material and to provide radiographic contrast relative to the low-density carbon foam payload for diagnostic purposes. Upon $\mathrm{X}$-ray absorption, the face of the bromine-doped plastic rapidly ablates and this ablation causes a strong shock wave to be launched into the experimental package.

Two beams of the Nova laser were used to generate $\mathrm{K}_{\alpha} \mathrm{X}$ rays of approximately $5 \mathrm{keV}$ by striking a chromium metal foil at the appropriate diagnostic time. The shock tube is backlit by these lasergenerated $\mathrm{X}$ rays and imaged with an $8 \mathrm{x}$ magnification $\mathrm{x}$-ray pinhole camera which provides an $\mathrm{X}$-ray radiograph of the sample. Radiographic contrast of the fluidized materials allows us to locate key features of the developing flow. Fiducial wires provide spatial calibration and reference locations. The $\mathrm{x}$-ray camera records the image on film which is subsequently scanned and digitized for analysis. Both the Flexible X-ray Imager (FXI) and Los Alamos Pinhole Camera (LAPC) have been separately fielded as the primary diagnostic for these experiments. The FXI is a gated instrument which provides separate, temporally-spaced images whereas the LAPC is not gated, but provides higher signal-tonoise levels. The shutter speed was $0.09 \mathrm{~ns}$ for the FXI, and $0.5 \mathrm{~ns}$ for the LAPC.

\section{NOVA EXPERIMENT SIMULATIONS}

To investigate the validity of the calculations, a series of Nova laser experiments has been simulated with CALE. The problem was run in a 2-D, axially symmetric format, which approximates the cylindrical geometry of the experimental package. The computational domain was resolved using 350 zones axially and 95 zones radially. The grid spacing is $5 \mu \mathrm{m}$ square everywhere for the first $1500 \mu \mathrm{m}$ 
of package length. The remaining $500 \mu \mathrm{m}$ at the downstream end of the shock tube uses a rectangular grid spacing where the radial dimension is held at $5 \mu \mathrm{m}$ but the axial dimension increases with a zoneto-zone ratio of 1.035 . The computational domain includes the beryllium shock tube, all interior materials, and a portion of gold which represents the hohlraum wall attachment region. Material and radiation are allowed to flow freely everywhere. Heating from the hohlraum is simulated by applying a time-dependent temperature source at the edge of the brominated plastic which is exposed to the hohlraum interior. Radiation transport is calculated using single-group diffusion.

A Planckian temperature source was derived by a Nova program team from a combination of Dante measurements, a series of shock break-out experiments using witness plates, and modeling by LASNEX. This temporal temperature distribution was then applied uniformly along the first k-line of the computational domain thus initiating the simulation. The time-dependent temperature source was further scaled by a multiplicative constant of 0.88 . This value was chosen from a previous computational/experimental campaign of a high speed jet flow which was conducted by A-Division. The high speed jet effort contains a large data set which employs the identical drive conditions as that used for the right-cylinder perturbation experiments. The temperature drive for the jet simulations were iterated to this data set such that the location of easily distinguishable features of the jet matched the simulation. We therefore believe that using the same multiplicative constant used for the jet experiments was the most objective way to scale the drive parameter for the right-cylinder perturbation experiments. A plot of the drive temperature history is shown in Fig. 2.

Table 1 lists pertinent material and shock parameters for the experiment. The Atwood number for the experiment is not a single number. This is due to pre-heat which causes the density to change before, and during shock transit through the material. We have thus listed a range of Atwood numbers.

\section{DISCUSSION}

A collection of simulations and Nova experiments has been assembled which dates back to the original work performed in July 1995. This work includes several obvious design iterations in addition to simulations that were not intended to be fielded as experiments but are of general interest. During the course of this work, the available laser drive energy was reduced. This undesirable reduction was unavoidable due to Nova operational requirements. Because of this, two drive 'types' were used. Both drives use the same characteristic Nova pulse shape, (06) PS1-01, which is a 1 ns square pulse. Drive type A refers to a nominal drive energy of $21.6 \mathrm{~kJ}$ whereas drive type $\mathrm{B}$ refers to a nominal drive energy of $18 \mathrm{~kJ}$. Note that the drive type refers only to Nova experimental data, not the simulated drive parameters.

At the present time, we do not have a CALE source for the reduced-energy drive. Adequately characterizing this reduced drive is beyond the scope of the present work and remains to be done. Here we simply employ the nominal drive source for all simulations. It is tempting to simply adjust the drive multiplier further to compensate for the reduced energy drive. As will be seen, however, some of the data which uses the reduced-energy drive indicates that while the shock location may match the simulation at a given time, it does not match the simulation later in time. Simply changing the drive multiplier would not correct this problem. The following is a summary of the work performed to date. Henceforth, we will indicate the run designation and the physicist's initials for the simulations, and the shot number for the Nova data for documentation purposes.

\section{i. null right-cylinder perturbation design, drive type $A$}

A single shot was fielded to determine the hydrodynamic behavior of the working materials in the absence of the right-cylinder perturbation. The initial material geometry, a CALE simulated radiograph, and the measured Nova radiograph are shown in Fig. 3. This design used a carbon foam payload at a density of $\rho=0.1 \mathrm{~g} / \mathrm{cm}^{3}$. The simulation at $\mathrm{t}=25 \mathrm{~ns}$ (run $\mathrm{c} 40 \mathrm{j}, \mathrm{KTC}$ ) indicates that the shock-compressed materials remain planar in the central region of the shock tube out to $200 \mu \mathrm{m}$ radius for the plastic, $250 \mu \mathrm{m}$ radius for the brominated plastic, and $335 \mu \mathrm{m}$ radius for the shock front in the carbon foam. Figure 3 also shows a measured Nova radiograph at $t=25$ ns (shot \#25091213) which 
has been scaled and aligned with the simulated radiograph for comparison purposes. The experimental data indicates a flat interface between the compressed materials, as desired, and a planar shock. In comparison to the simulation however, the data shows approximately $300 \mu \mathrm{m}$ more compression between the brominated plastic and the shock in the axial direction. We believe the most likely explanation for this discrepancy is the inaccurate modeling of the hohlraum drive. As will be shown below, this discrepancy is evident in the majority of our comparisons.

\section{ii. $200 \mu \mathrm{m} \times 200 \mu \mathrm{m}$ right-cylinder perturbation, drive type $A$}

This design is the "nominal" configuration and it refers to a $200 \mu \mathrm{m}$ diameter x $200 \mu \mathrm{m}$ long rightcylinder perturbation and $\rho=0.1 \mathrm{~g} / \mathrm{cm}^{3}$ carbon foam payload. Figure 4 shows a $\Delta \mathrm{t}=5.0 \mathrm{~ns}$ temporal sequence out to time $\mathrm{t}=40 \mathrm{~ns}$ of the flow in which the materials are shown shaded. The simulation (run c40a, KTC) indicates that the right-cylinder perturbation itself lags behind the ensuing material which flows around the perturbation in the outer regions. This shear causes a small amount of rollup of material. A core region of carbon foam remains within the perturbation, even at late times. Figure 5 shows simulated radiographs of this flow with material boundaries indicated for illustrative purposes, and Fig. 6 shows a comparison of the simulations with Nova data. Two diagnostic times were chosen, $\mathrm{t}=25 \mathrm{~ns}$ and $\mathrm{t}=40 \mathrm{~ns}$, because of the distinguishing features present at these times. Comparison at $\mathrm{t}=25 \mathrm{~ns}$ (shot \# 25072019) indicates that both the width and length of the rightcylinder perturbation is in good agreement, as is vortex development at the sides of the perturbation. The agreement is worse for the spacing of the shock front and the perturbation center, in which the experiment is about $60 \mu \mathrm{m}$ smaller. Similarly, the locations of the brominated and plain plastic behind the perturbation are in poor agreement. Comparison at $40 \mathrm{~ns}$ indicates further discrepancy of the brominated plastic layer. The simulation shows a core of brominated plastic confined approximately to the inner diameter of the shock tube, which follows behind the perturbation. The data, however, (shot \# 25091215) shows the brominated plastic wrapping around the perturbation in a semi-circle shape. Further, the edges of the brominated plastic trail downstream in a wing-like pattern. The data appears more circular than the simulation, and the thickness of the plain plastic layer is substantially reduced. These comparisons consistently indicate that the simulation is not driven to the same degree of compression as that observed experimentally.

\section{iii. $200 \mu m \times 200 \mu m$ right-cylinder perturbation with pencil tracer, drive type $A$}

Figure 7 shows a configuration that is referred to as the pencil tracer design. The initial geometry uses the nominal right-cylinder perturbation design, but a $200 \mu \mathrm{m}$ diameter $\mathrm{x} 700 \mu \mathrm{m}$ long $\mathrm{SiO}_{2}$ aerogel tracer is placed adjacent and downstream of the perturbation. The tracer material is density-matched with the surrounding foam at $\rho=0.2 \mathrm{~g} / \mathrm{cm}^{3}$. This density was chosen because fabrication of the tracer using $\rho=0.1 \mathrm{~g} / \mathrm{cm}^{3}$ material was not possible. Figure 7 shows the initial tracer material before insertion into a hole drilled in the carbon foam payload. The tracer material was designed to trace a hydrodynamic vortex ring. Inspection of the hydrodynamic evolution of the nominal right-cylinder perturbation design indicates that the perturbation disrupts the advancing shock such that after the shock has passed through the perturbation, the merging of the shock generates baroclinic vorticity which forms a vortex ring. This vortex ring travels downstream ahead of the perturbation and in close proximity to the shock, which retains a distorted shape. Simulations at $t=32.5 \mathrm{~ns}$ (run $\operatorname{lmlp} 4, \mathrm{LML}$ ) indicate that the tracer material is collected by the vortex ring which rides just behind the shock front while a core of on-axis material remains connected to the perturbation material. The Nova data (shot \# 27032021) indicates a diffuse radiograph in which the dimensions of the vortex rollup are indiscernible. The location of the shock agrees well with the simulation. However, the data indicates greater on-axis compression and a "wing-shaped" brominated plastic layer which is not observed in the simulations. An additional simulation (run lmlp5, LML) and experiment (shot \# 27061208) were performed without the right-cylinder perturbation in order to observe the behavior of the tracer material in its absence. The geometry was similar to that shown in Fig. 7 with the exception that perturbation material was replaced with additional tracer material making the pencil tracer $900 \mu \mathrm{m}$ long. The simulation shows that the tracer compresses in the axial direction to about $170 \mu \mathrm{m}$ long and expands to about $300 \mu \mathrm{m}$ diameter. The data does not show a sufficiently resolved image of the tracer for comparison but it does indicate greater on-axis compression, a wing-shaped brominated plastic layer, 
and the shock location advanced by $70 \mu \mathrm{m}$. The pencil tracer design was ultimately unsuccessful in determining vortex geometry and structure.

\section{iv. $100 \mu m \times 200 \mu m$ right-cylinder perturbation, drive type $A$}

Two shots were fielded with a $100 \mu \mathrm{m}$ diameter $\mathrm{x} 200 \mu \mathrm{m}$ long right-cylinder perturbation and $\rho=0.1$ $\mathrm{g} / \mathrm{cm}^{3}$ carbon foam. The initial geometry, simulations (run $\mathrm{c} 44 \mathrm{r}, \mathrm{KTC}$ ), and data are shown in Fig. 8 at $\mathrm{t}=25 \mathrm{~ns}$ (shot \# 25082214) and $\mathrm{t}=30 \mathrm{~ns}$ (shot \# 25082217). Comparison of simulation and data indicates a substantial discrepancy, especially with regard to the brominated plastic ablator. The simulation shows the ablator expanding outwards near the hohlraum end and tapering down to the inside shocktube diameter. The data, however, shows the ablator to be highly compressed into a wing shape, with the wing tips turning upstream abruptly near the ends. The data shows that the ablator is further compressed with increasing time, whereas the simulation indicates a slight expansion of the ablator. In the simulation at $\mathrm{t}=30 \mathrm{~ns}$, the brominated plastic layer is approximately $580 \mu \mathrm{m}$ thick however the data is approximately $150 \mu \mathrm{m}$ thick. Fine details of the right-cylinder perturbation are not visible in the data but at both diagnostic times, the perturbation looks more compressed than that indicated by the simulation, being nearly as long as it is wide. The simulation shows the perturbation to be elongated in the axial direction. Although the shock is approximately in the correct location in comparison to the simulation, the increased compression of the perturbation and brominated plastic makes the agreement extremely poor.

\section{V. $200 \mu \mathrm{m} \times 200 \mu \mathrm{m}$ right-cylinder perturbation, drive type $B$}

Figure 9 shows simulations (run c40b, KTC) and data (shot \#28063015, shot \# 28071009) using the nominal right-cylinder perturbation design with the reduced energy drive type $B$, and $\rho=0.2 \mathrm{~g} / \mathrm{cm}^{3}$ carbon foam. The increased foam density was used to make the shock more visible because its structure downstream of the perturbation is of interest. Comparisons were made at $\mathrm{t}=25$ and $\mathrm{t}=40$ ns. The simulations indicate that the larger foam density reduces the shock and flow velocities. The perturbation length appears reduced in comparison to the data used in Fig. 6, but the data shows an even more compressed (by about $100 \mu \mathrm{m}$ ) perturbation. The shock front has less curvature in these simulations compared to Fig. 6, but this could be an artifact of the slower flow development. Comparison shows the shock front to be approximately $200 \mu \mathrm{m}$ downstream of that seen in the data. This is most likely due to the reduced drive energy of the experiment relative to the CALE drive which was not designed for the type B drive conditions. In general, the data again indicates a compressed perturbation, a compressed brominated plastic layer (especially at $\mathrm{t}=40 \mathrm{~ns}$ ), and a reduced ablator-toshock distance in comparison to the simulations.

\section{vi. $400 \mu m \times 200 \mu m$ right-cylinder perturbation, drive type $B$}

Figure 10 shows a revised geometry in which the right-cylinder perturbation diameter was increased to $400 \mu \mathrm{m}$ while the perturbation length was maintained at $200 \mu \mathrm{m}$ and a $\rho=0.2 \mathrm{~g} / \mathrm{cm}^{3}$ carbon foam was used. Comparison of simulations (run c40d, KTC) and data were made at $t=25$ and $t=40 \mathrm{~ns}$. At $t$ $=25 \mathrm{~ns}$ the perturbation looks compressed slightly more in the simulation than in the data, but the diameter is approximately correct. In the data at $t=25 \mathrm{~ns}$ (shot \#28073021), we can just resolve rollup on both sides of the perturbation, and the hint of a center jet-like feature. Both of these features are clearly visible in the simulation. The data shows a cusp-shaped shock which is also visible in the simulation and in the correct location. The data indicates that the brominated plastic rides closer to the perturbation than that observed in the simulation. In the simulation, the brominated plastic is behind the perturbation by as much as $200 \mu \mathrm{m}$, whereas the data indicates a maximum of $120 \mu \mathrm{m}$. At $\mathrm{t}=40$ $\mathrm{ns}$, the simulation indicates that the rollups become more developed, the center jet appears to be longer, and the shock front has a convex shape. The data (shot \# 28081311) shows the perturbation to be compressed $75 \mu \mathrm{m}$ more than the simulation, and the brominated plastic region to be within $50 \mu \mathrm{m}$ of the perturbation, compared to $360 \mu \mathrm{m}$ for the simulation. The shock has a slight convex shape, and its location is approximately $200 \mu \mathrm{m}$ behind that observed in the simulation. The advanced shock position seen in the simulation could allow additional freedom for the development of the on-axis jet which is not as visible in the data. The close proximity of the ablator to the perturbation seen in the data indicates a much larger back pressure than that seen in the simulations. Overall, the right-cylinder 
perturbation features correlate well between the simulations and the data, but the exact positions and thickness of the other materials differ.

\section{vii. clear $C H$ design, drive type $B$}

Simulations have been performed for a design which incorporates the standard $200 \mu \mathrm{m}$ diameter $\mathrm{x} 200$ $\mu \mathrm{m}$ long brominated plastic right-cylinder perturbation $\left(\rho=1.57 \mathrm{~g} / \mathrm{cm}^{3}\right)$ surrounded by a layer of plastic $\left(\rho=1.05 \mathrm{~g} / \mathrm{cm}^{3}\right)$ which extends to the inside diameter of the shock tube, and a $\rho=0.2 \mathrm{~g} / \mathrm{cm}^{3}$ carbon foam payload. The initial geometry is shown in Fig. 11. Simulations at $t=25 \mathrm{~ns}$ and $40 \mathrm{~ns}$ are also shown (run $\mathrm{c} 41 \mathrm{~m}, \mathrm{KTC}$ ). This configuration allows us to view the core region of the rightcylinder perturbation while the hydrodynamic flow around the perturbation is blocked. At $t=25 \mathrm{~ns}$ the simulation shows the core of the perturbation to have a jet-like shape. The brominated plastic region does not travel as far and remains planar because it is blocked by the plain plastic material around the perturbation, and cannot flow freely around it. The shock is only slightly ahead of the perturbation, and is distorted by the perturbation flow. The shock contains a large bump at $\mathrm{t}=25 \mathrm{~ns}$ about $80 \mu \mathrm{m}$ further ahead of the flat shock portion. At $t=40 \mathrm{~ns}$ the perturbation continues to behave like a jet in which the on-axis core becomes quite long, approaching $400 \mu \mathrm{m}$ in length. The brominated plastic layer remains planar, and the jet tip remains along with the advancing shock. The distortion of the shock is not as noticeable at this later time. The perturbation itself takes on a small rollup feature which grows in length with time. The rollup has not completed one full cycle, and appears relatively stable.

\section{viii. slab tracer design, drive type $B$}

Figure 12 shows an additional target type which uses the nominal right-cylinder perturbation design with a slab tracer layer made of brominated plastic located downstream at $1100 \mu \mathrm{m}$. The slab is $50 \mu \mathrm{m}$ thick. The tracer layer is designed to highlight the vortex ring produced by baroclinic vorticity rollup from the main shock. The flowfield will not be altered until shock arrival at the tracer, at which time the existence and diameter of the vortex structure should be observable. Different tracer layer thickness were studied and this design was found to best highlight the vortex feature. We chose brominated plastic as the tracer for ease of construction. In the simulation at $\mathrm{t}=24 \mathrm{~ns}$ (run c44p, KTC), a distinct vortical feature can be seen breaking through the shock-compressed tracer layer. However, this feature may not be distinctly observable in a Nova experiment. The simulations also indicate that the data acquisition timing will be critical to the success of the experiment as the temporal duration of the observability of the feature is limited to approximately $1 \mathrm{~ns}$.

\section{ix. additional simulations}

Two additional simulations were performed in which the nominal $200 \mu \mathrm{m}$ diameter $\mathrm{x} 200 \mu \mathrm{m}$ long right-cylinder perturbation design was modified by changing the density of the carbon foam material from $\rho=0.1 \mathrm{~g} / \mathrm{cm}^{3}$ to $\rho=0.01 \mathrm{~g} / \mathrm{cm}^{3}$, and $\rho=1.0 \mathrm{~g} / \mathrm{cm}^{3}$. These changes allow for a quick assessment of the hydrodynamic dependence on the Atwood number. In Fig. 13 (a), the density contours for the low-density $\left(\rho=0.01 \mathrm{~g} / \mathrm{cm}^{3}\right)$ configuration is shown (run lmlp1, LML). In this case the foam is seen to provide less resistance to the material at the outer edges of the right-cylinder perturbation, and this material jets downstream rapidly. The high speed flow results in lower material pressures which allows the perturbation to expand. The shock appears only slightly perturbed. Figure 13 (b) represents the nominal design with a foam density of $\rho=0.1 \mathrm{~g} / \mathrm{cm}^{3}$ and is included for comparison purposes (run lmlp3, LML). In this case the perturbation material is substantially deformed and accelerated. The shock is perturbed by the on-axis vortex structure mentioned previously. In Fig. 13 (c) the foam density is increased to $\rho=1.0 \mathrm{~g} / \mathrm{cm}^{3}$ (run $1 \mathrm{mlp} 3$, LML). The shock speed is seen to be much slower in this case. There is some indication of perturbation rollup, but it is substantially reduced from previous cases. Decreasing (increasing) the foam density increases (decreases) the Atwood number, or density contrast. Decreasing the Atwood number reduces vorticity production at the density interface, as shown in Fig. 13 (c). A smaller Atwood number also reduces the effect of Rayleigh-Taylor mixing. 


\section{CONCLUSIONS}

This work indicates that CALE is a useful tool for investigating the hydrodynamic behavior of shocked materials. Simulations of the Nova right-cylinder perturbation targets, however, consistently misrepresent the amount of compression which is observed experimentally. The increased compression of the data in comparison to simulations is most likely due to errors in the modeling of the drive conditions, though it is possible that it is caused by problems in our set-up deck or even the code algorithms.

\section{ACKNOWLEDGMENTS}

This work benefited greatly from previous efforts by T. Perry, K. Budil, P. Stry, and P. Miller. We would like to also acknowledge the skilled target fabrication efforts of Hedley Louis, Tony Demiris, and their group of machinists, contributions by Russell Wallace, the work of Nova target assembly and Nova operations, and the technical assistance of Andy Nikitin, Steve Compton, and Vern Rekow. This work was performed under the auspices of the U.S. Department of Energy by Lawrence Livermore National Laboratory under contract W-7405-ENG-48. 


\begin{tabular}{|c|c|}
\hline Pat & $\begin{array}{l}\text { Noya Experiment } \\
\text { (cHBr in Carbon foam) }\end{array}$ \\
\hline \multicolumn{2}{|l|}{ Perturbation } \\
\hline Size & $0.1-0.75 \times 0.2 \mathrm{~mm}$ \\
\hline Initial temperature (pre-heat) & $0.5 \mathrm{eV}$ \\
\hline Atwood number (pre-shock) & -0.83 to -0.88 \\
\hline Sound Speed (pre-heat) & $6 \mu \mathrm{m} / \mathrm{ns}$ \\
\hline \multicolumn{2}{|l|}{ Parameters at perturbation } \\
\hline$\Delta \mathrm{v}_{\text {shook }}$ & $40 \mu \mathrm{m} / \mathrm{ns}$ \\
\hline$\Delta \mathrm{P}_{\text {shock }}^{\text {shock }}$ & $20 \mathrm{Mbar}$ \\
\hline Mach number & 6.4 \\
\hline $\mathrm{I}\left(\Delta \mathrm{P} / \mathrm{\rho}\right.$-sound speed $\left.{ }^{2}\right)$ & 33.6 \\
\hline Material temperature (post-shock) & $2-3 \mathrm{eV}$ \\
\hline Re (estimated, $-\mathrm{L} / \mathrm{ion} \mathrm{m} . \mathrm{p}$ ) & $8 \times 10^{4}$ \\
\hline Se (estimated, V/D) & 136 \\
\hline
\end{tabular}

${ }^{*}$ Atwood Number $=\frac{\rho_{2}-\rho_{1}}{\rho_{2}+\rho_{1}}, \quad \rho_{1}=\rho_{\text {right-cylinder persurbation }}, \rho_{2}=\rho_{\text {carbon foum }}$

Table 1. The Nova experiment parameters. 

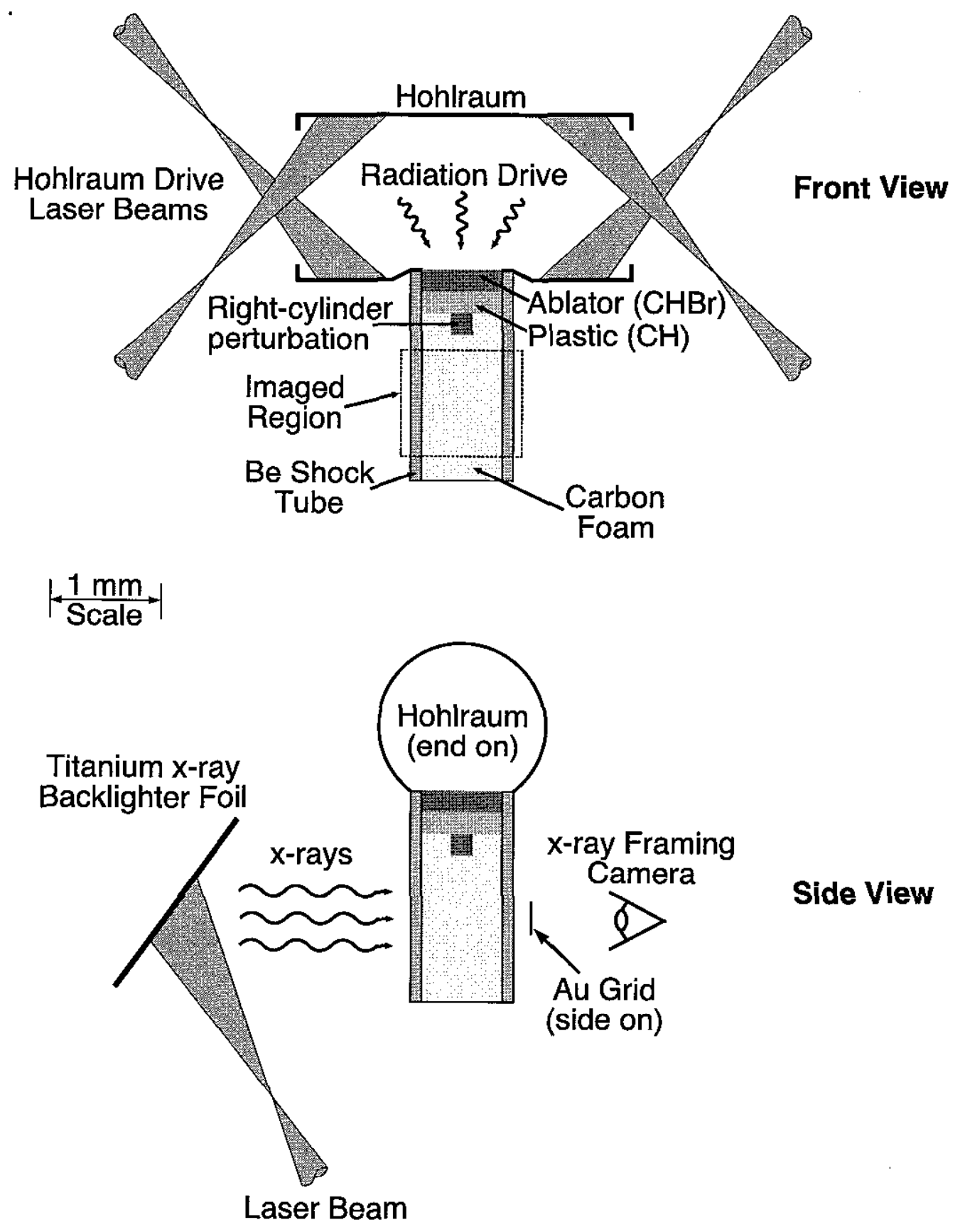

Figure 1. Front and side view of the experimental geometry. A right-cylinder perturbation is located at the plastic/carbon-foam interface. 


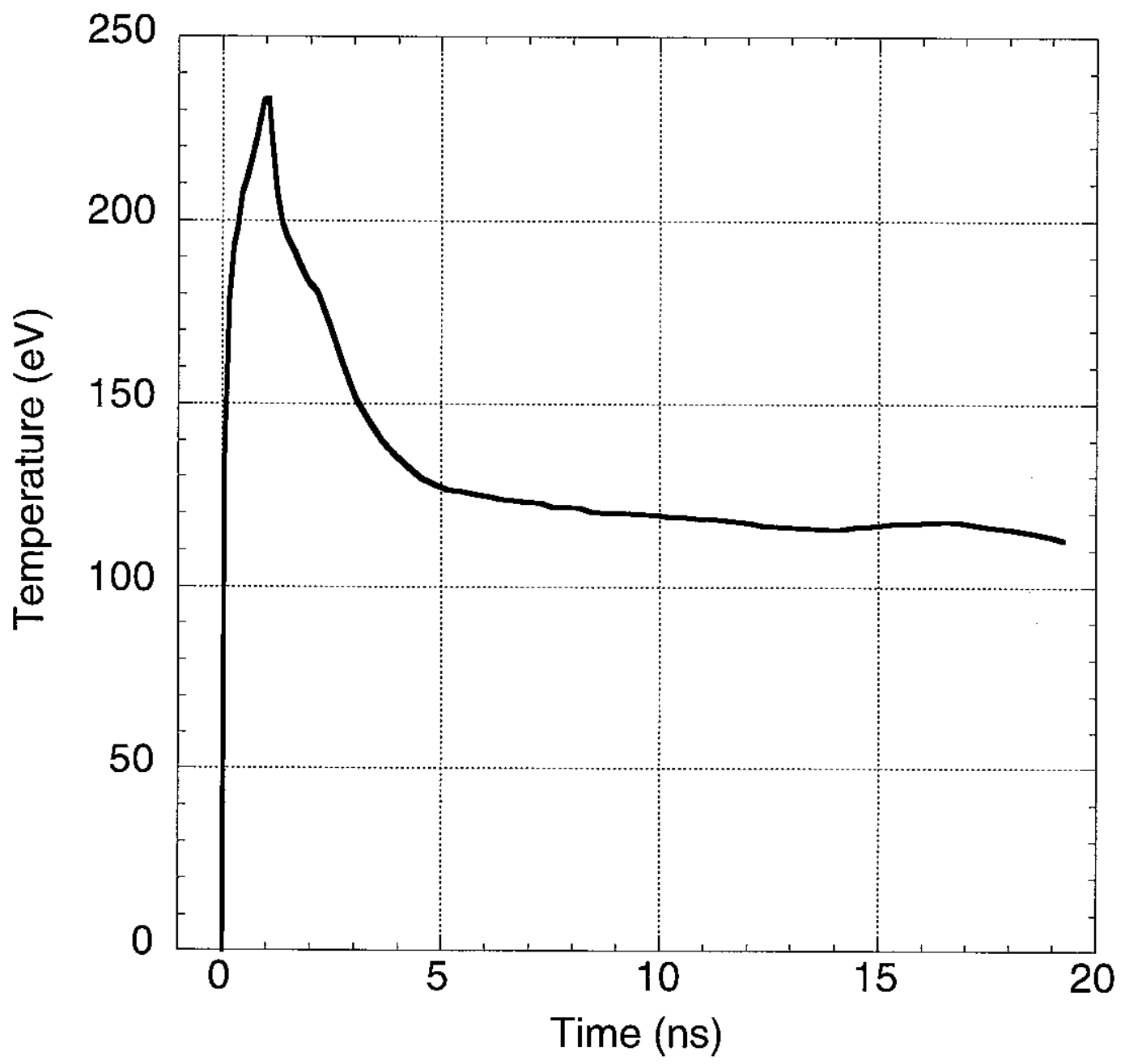

Figure 2. Hohlraum drive used for the simulations. 

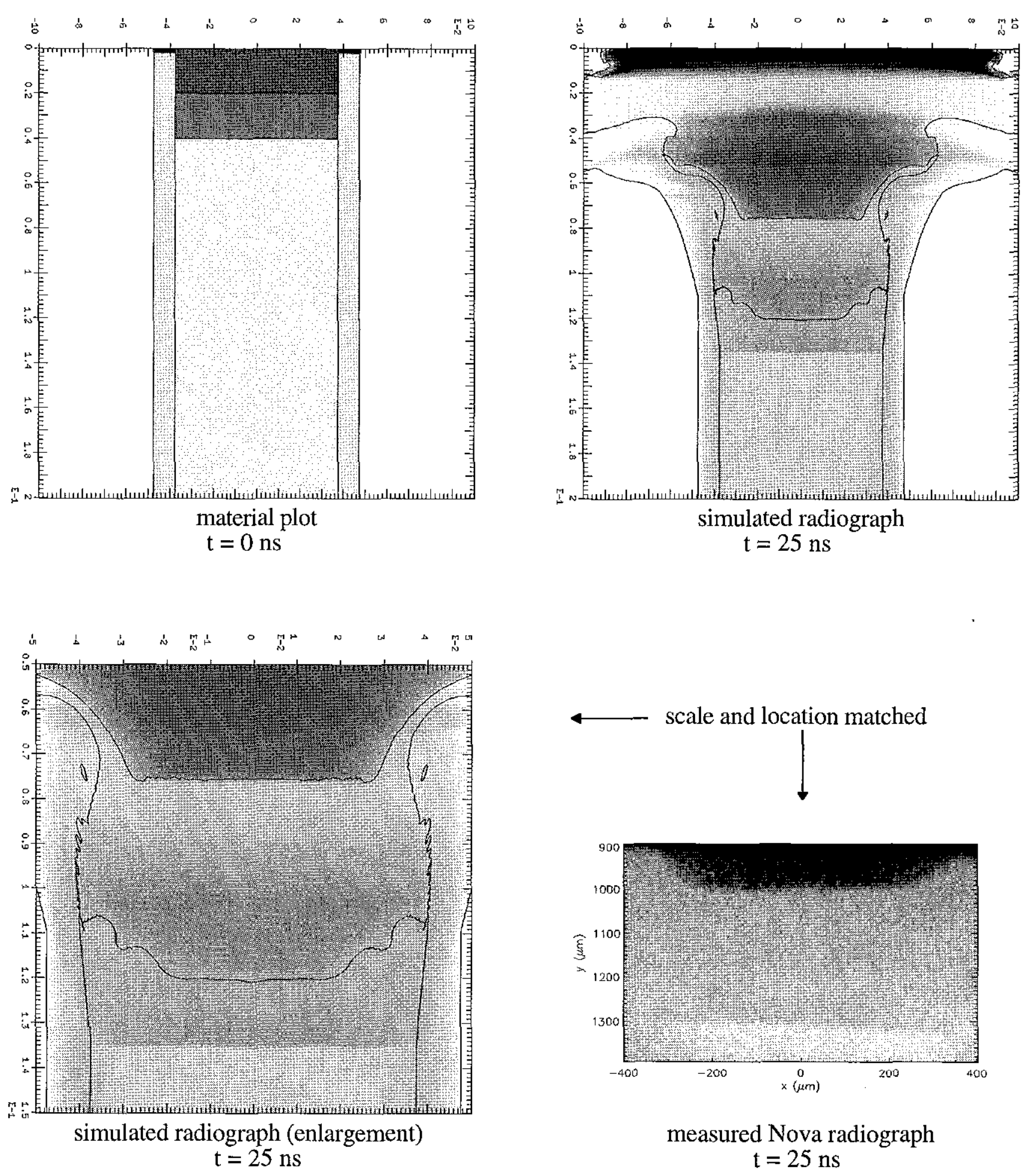

Figure 3. The null right-cylinder perturbation design employing drive type A. Comparison of simulated and experimental radiographs at $\mathrm{t}=25 \mathrm{~ns}$ indicates that the distance between the brominated plastic and the shock is $300 \mu \mathrm{m}$ smaller in the Nova data than that indicated in the simulation. 

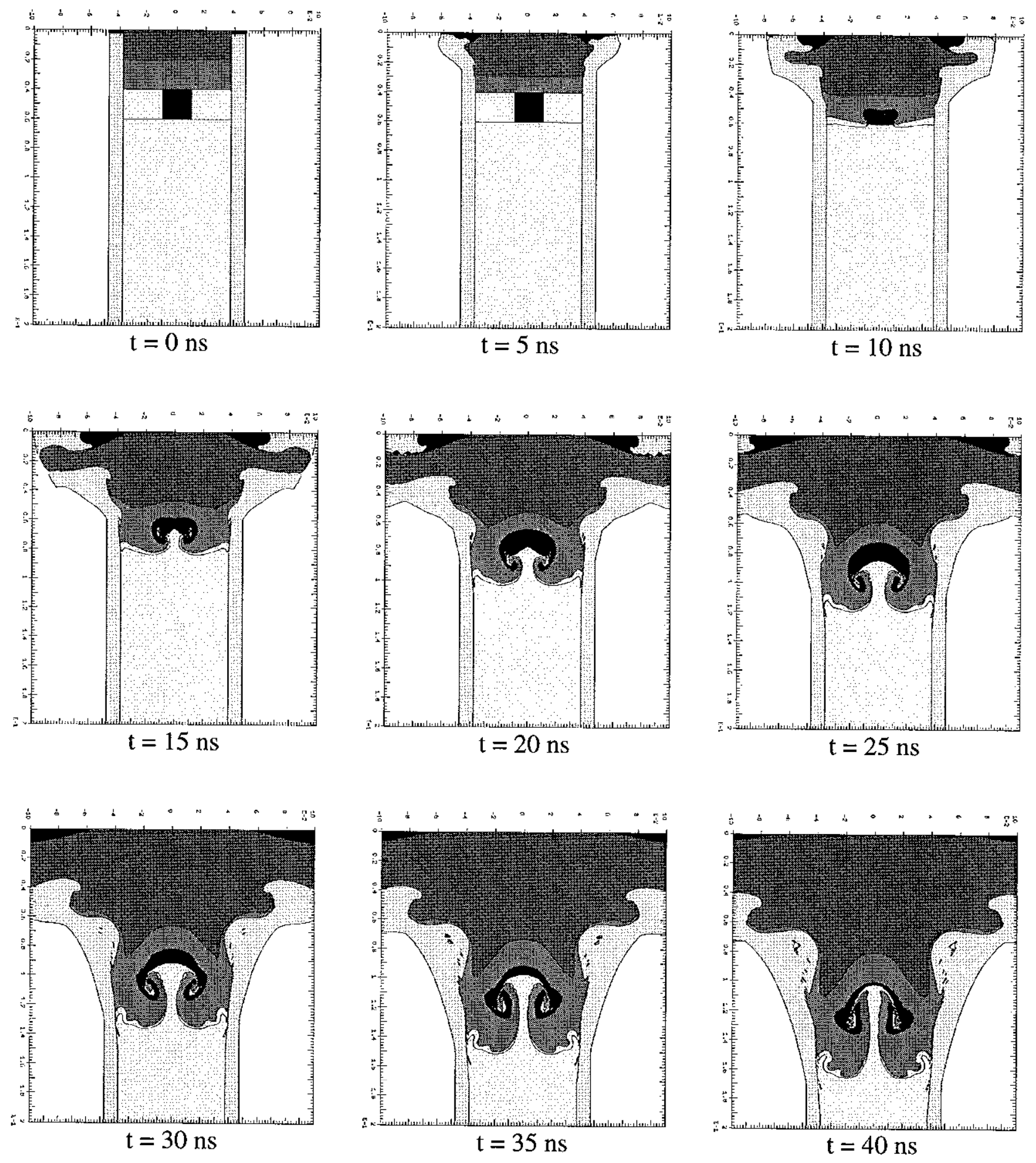

Figure 4. Temporal sequence of the flowfield with a $200 \mu \mathrm{m}$ diameter $\mathrm{x} 200 \mu \mathrm{m}$ long right-cylinder perturbation. The different materials in the flow are depicted with various shades of gray. 

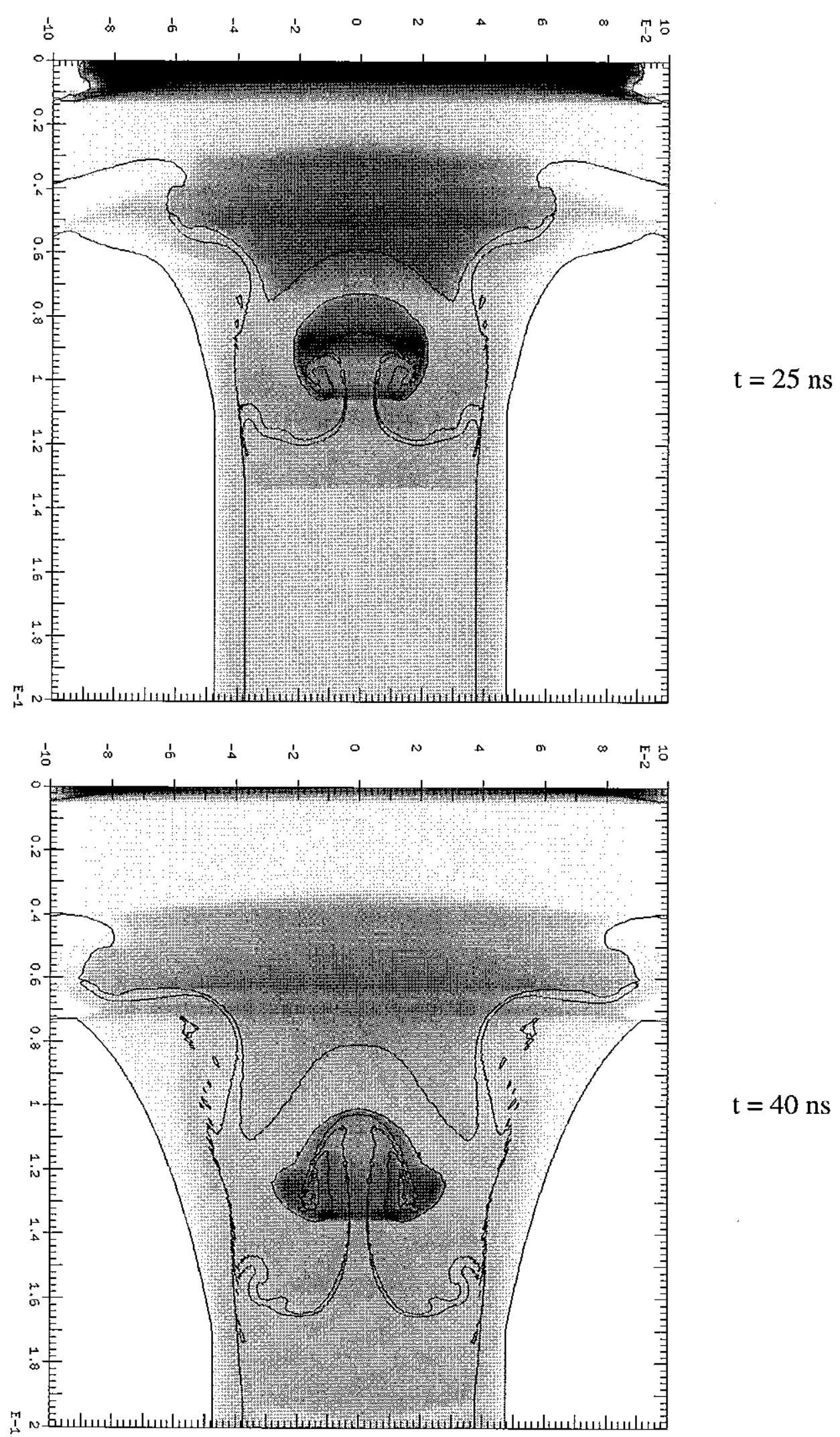

Figure 5. Simulated radiographs of the $200 \mu \mathrm{m}$ diameter $\mathrm{x} 200 \mu \mathrm{m}$ long right-cylinder perturbation at $\mathrm{t}$ $=25 \mathrm{~ns}$ and $\mathrm{t}=40 \mathrm{~ns}$. 

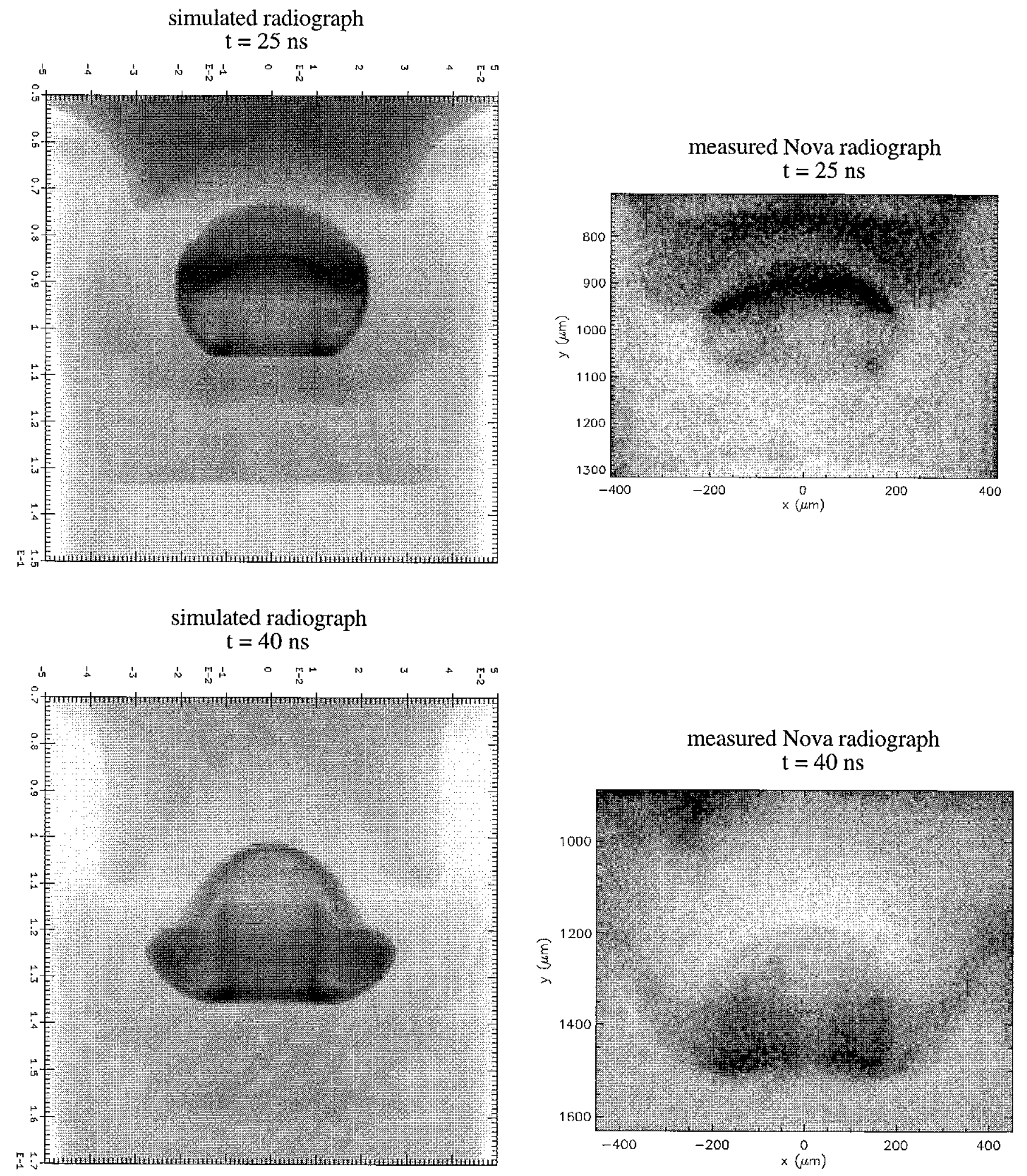

Figure 6. Comparison of simulated and experimental radiographs at $t=25 \mathrm{~ns}$ and $\mathrm{t}=40 \mathrm{~ns}$ for a 200 $\mu \mathrm{m}$ diameter $\mathrm{x} 200 \mu \mathrm{m}$ long right-cylinder perturbation using drive type $\mathrm{A}$. 


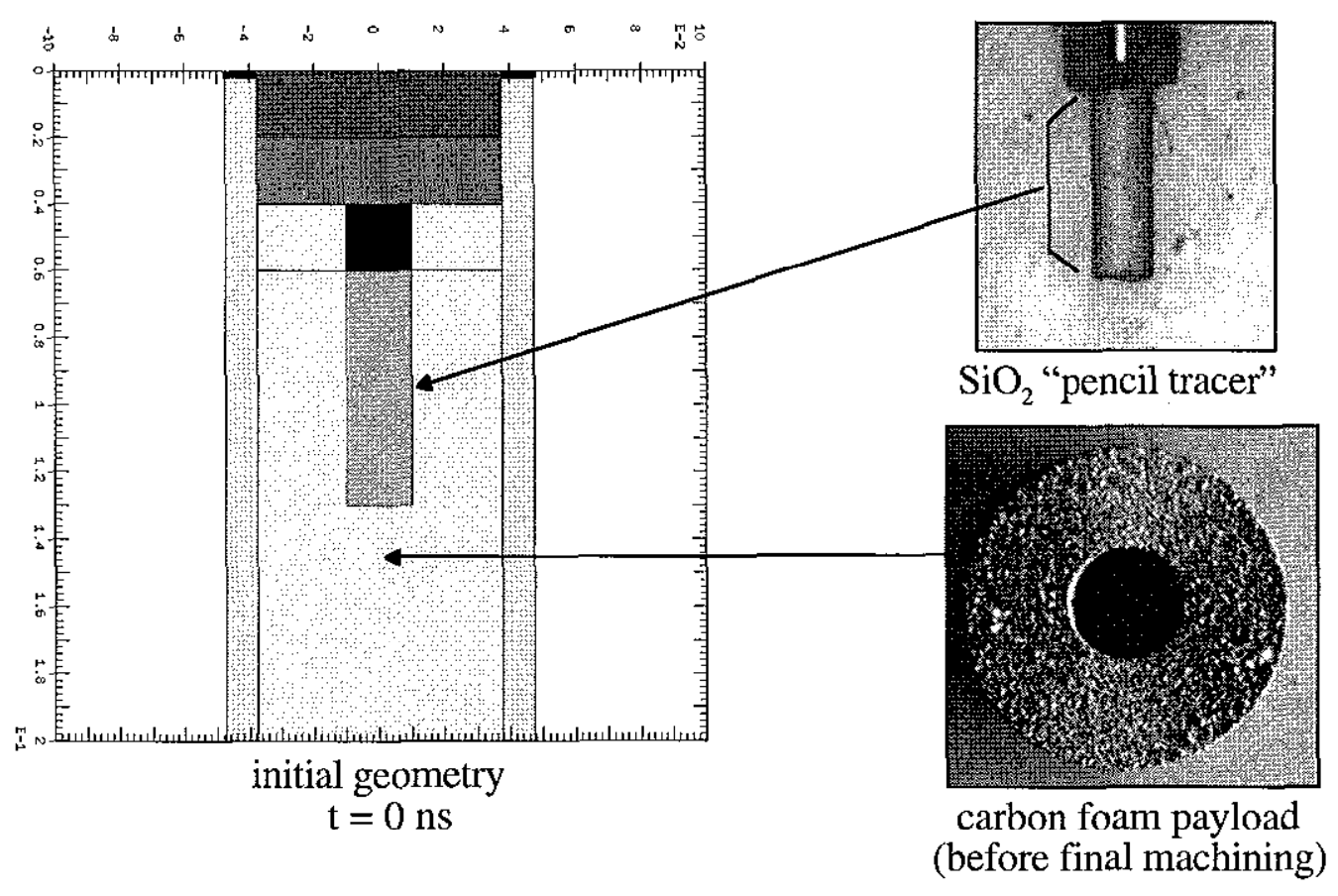

simulated
radiograph
$t=32.5 \mathrm{~ns}$
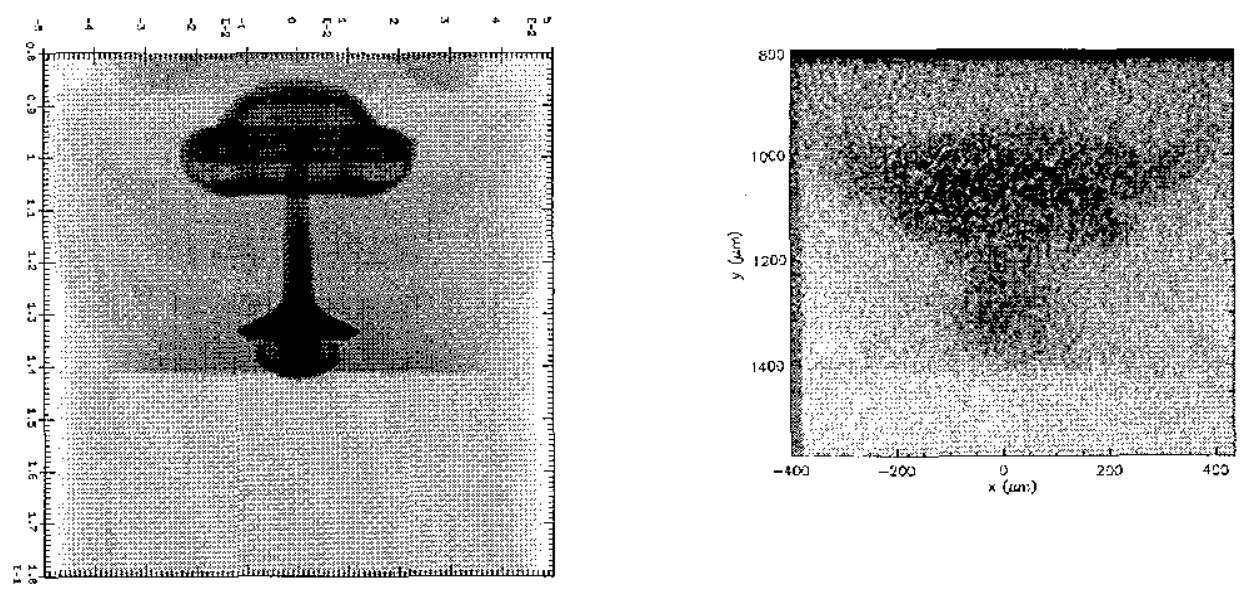

Nova data

$\mathrm{t}=32.5 \mathrm{~ns}$

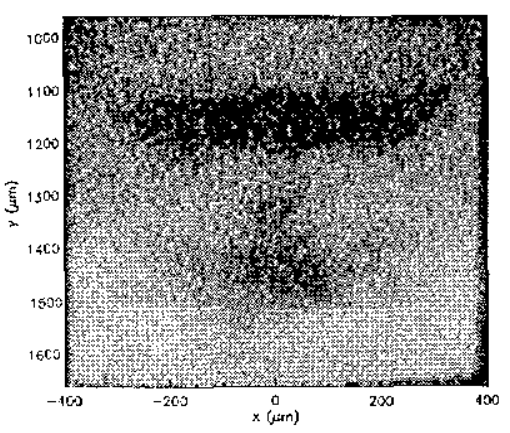

Nova data

$\mathrm{t}=32.5 \mathrm{~ns}$

- no right-

cvlinder

Figure 7. Pencil tracer design. The initial geometry employs a pencil-shaped $200 \mu \mathrm{m}$ diameter $\mathrm{x} 700$ $\mu \mathrm{m}$ long $\mathrm{SiO}_{2}$ aerogel tracer that is density-matched with the surrounding foam $\left(\rho=0.2 \mathrm{~g} / \mathrm{cm}^{3}\right)$ and placed adjacent to the right-cylinder perturbation. 


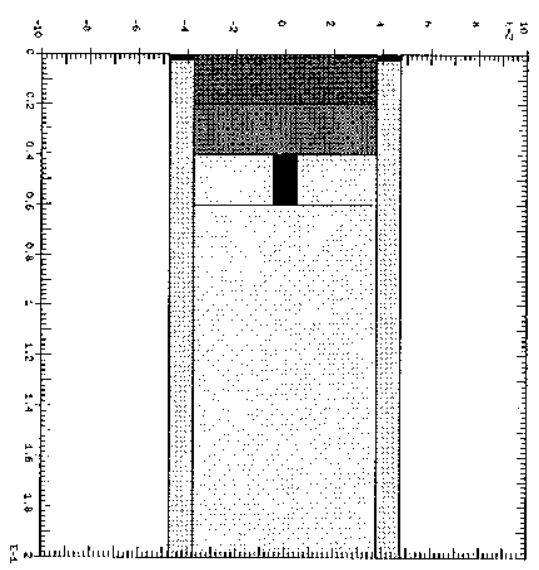
initial geometry $\mathrm{t}=0 \mathrm{~ns}$

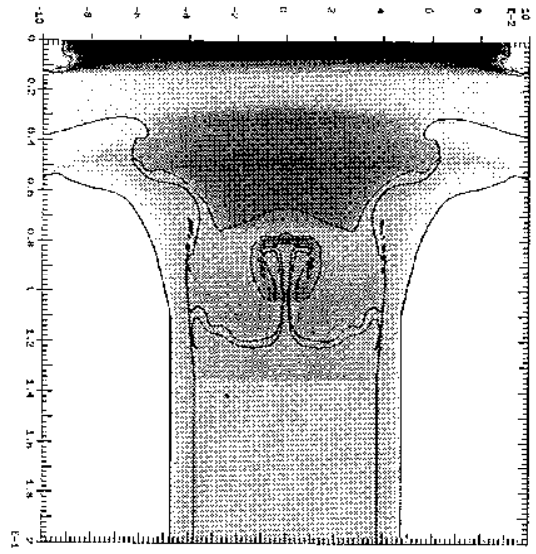

simulated radiograph

$\mathrm{t}=25 \mathrm{~ns}$

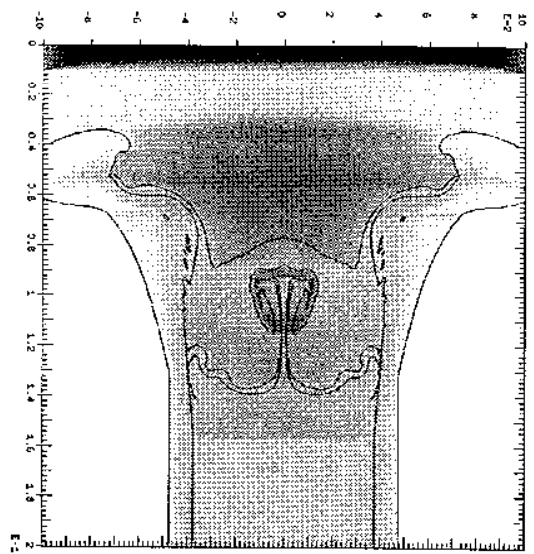

simulated radiograph $\mathrm{t}=30 \mathrm{~ns}$
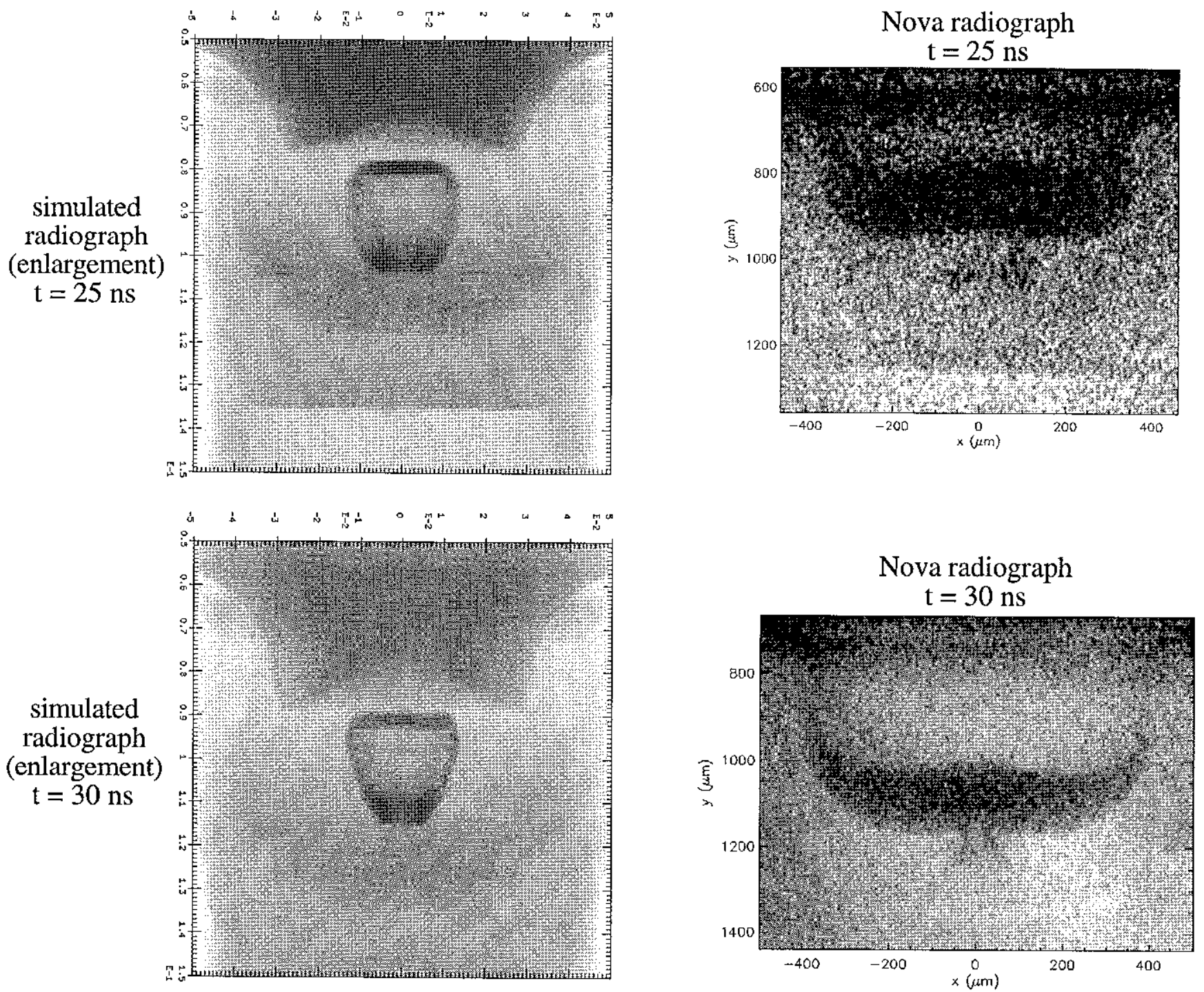

Figure 8. The $100 \mu \mathrm{m}$ diameter $\mathrm{x} 200 \mu \mathrm{m}$ long right-cylinder perturbation design employing drive type A. Comparison of simulated and experimental radiographs at $t=25$ and 30 ns indicates several discrepancies. 

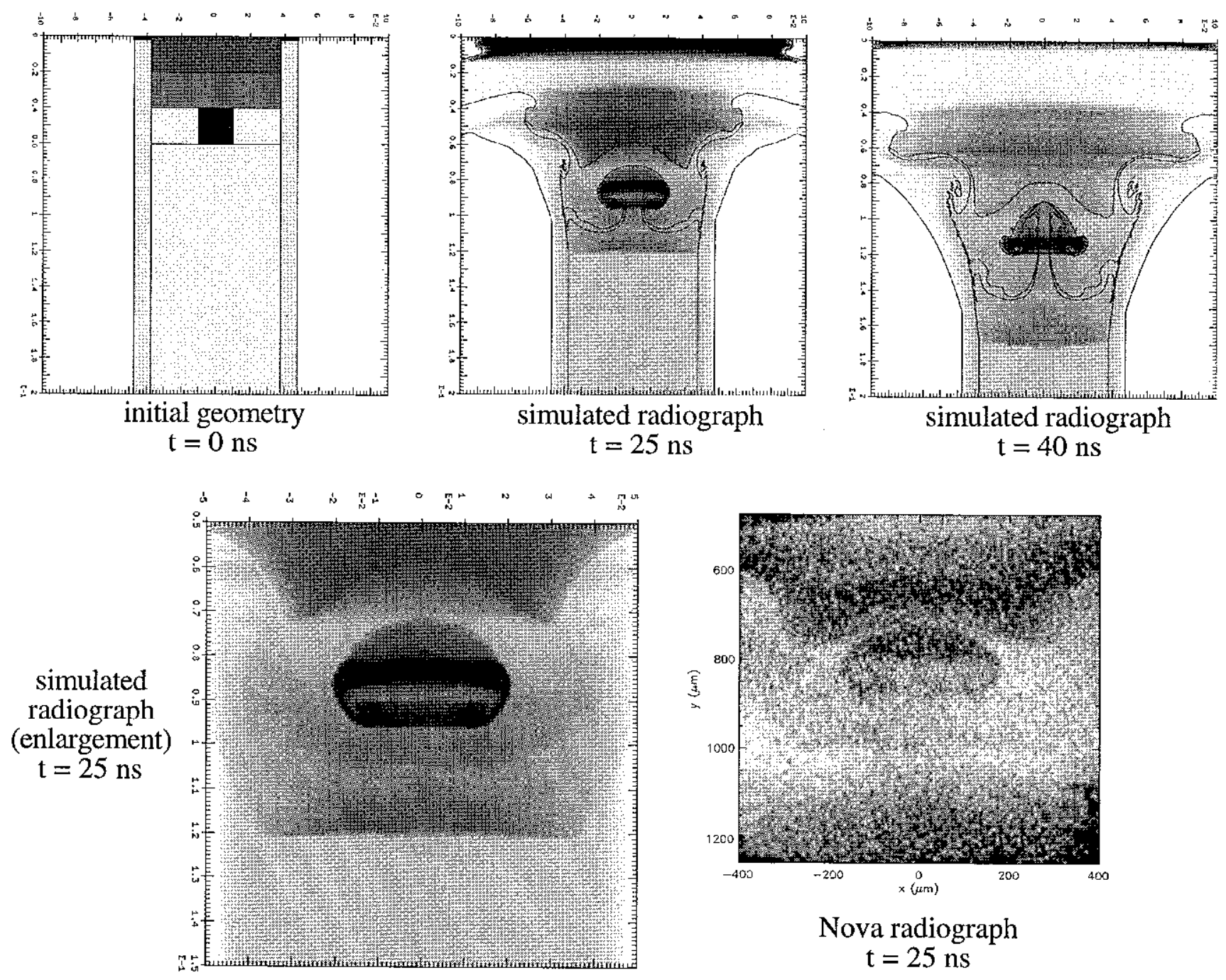

Nova radiograph

$\mathrm{t}=25 \mathrm{~ns}$
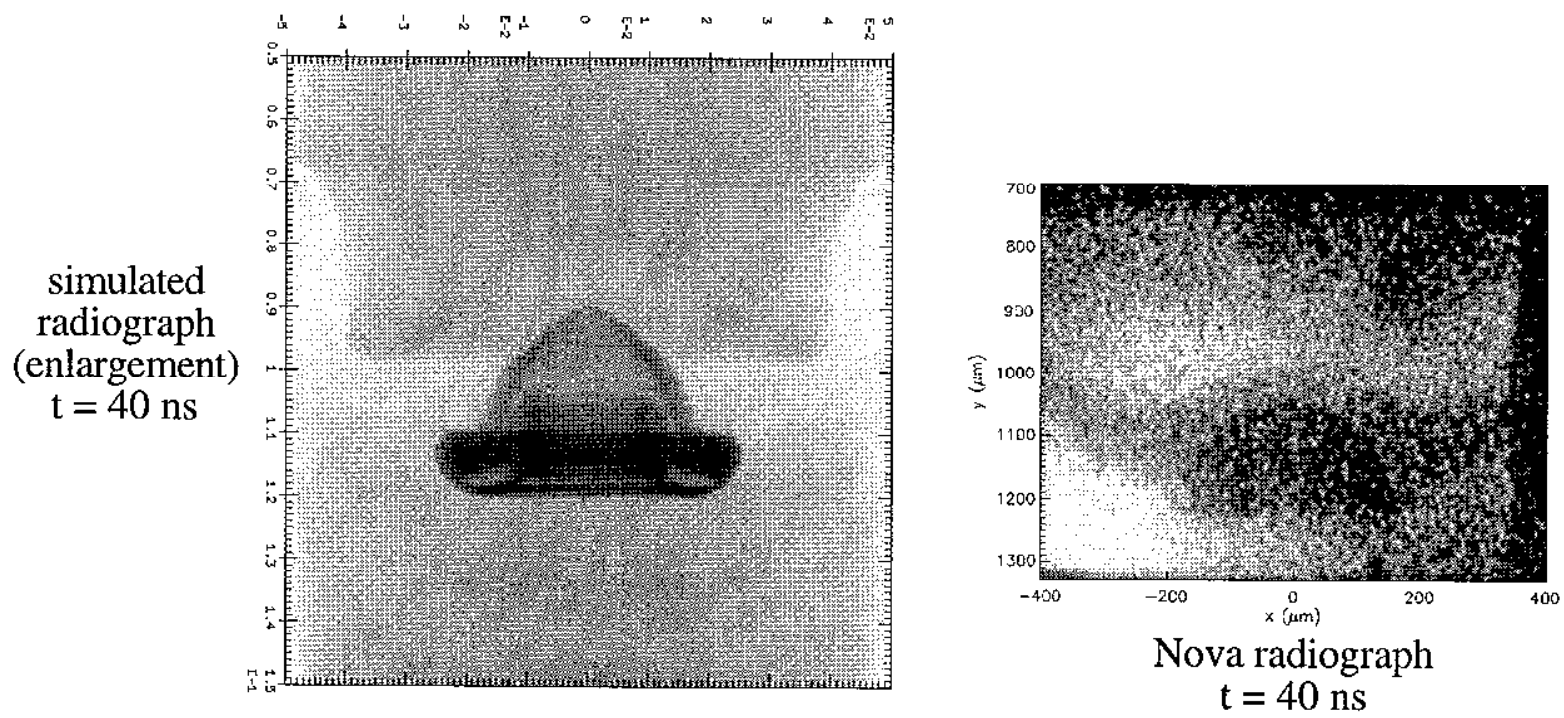

Figure 9. The $200 \mu \mathrm{m}$ diameter $\times 200 \mu \mathrm{m}$ long nominal right-cylinder perturbation design using the reduced-energy drive type $B$. Comparisons of simulated and experimental radiographs at $t=25 \mathrm{~ns}$ and $\mathrm{t}=40 \mathrm{~ns}$ are shown. 

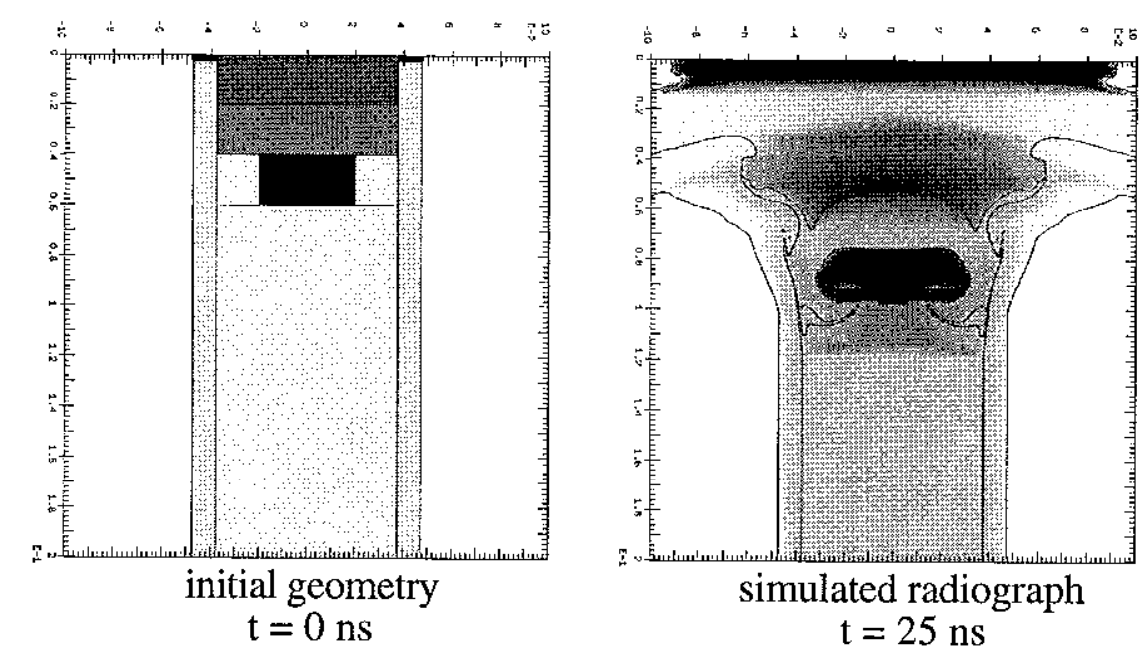

$\mathrm{t}=25 \mathrm{~ns}$
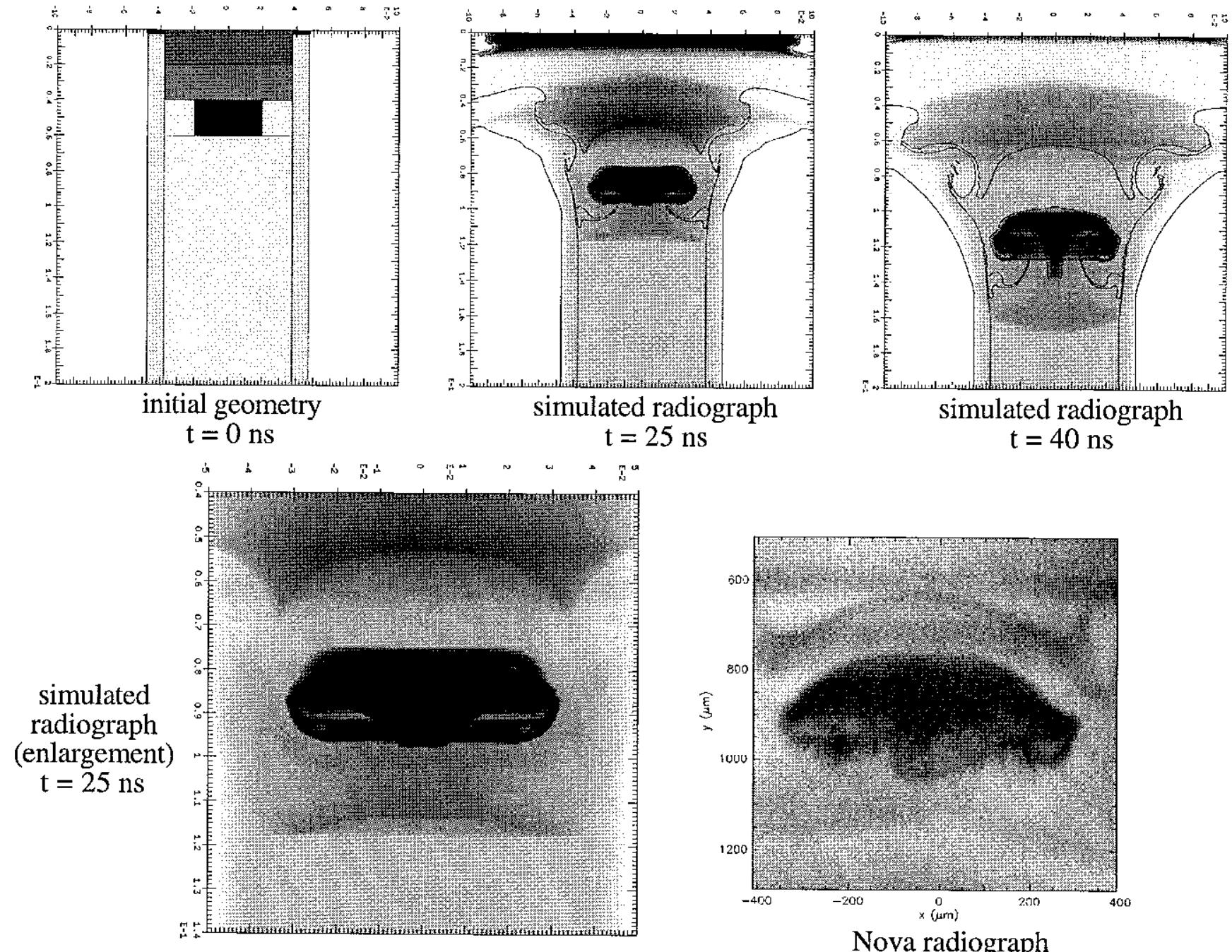

Nova radiograph

$\mathrm{t}=25 \mathrm{~ns}$
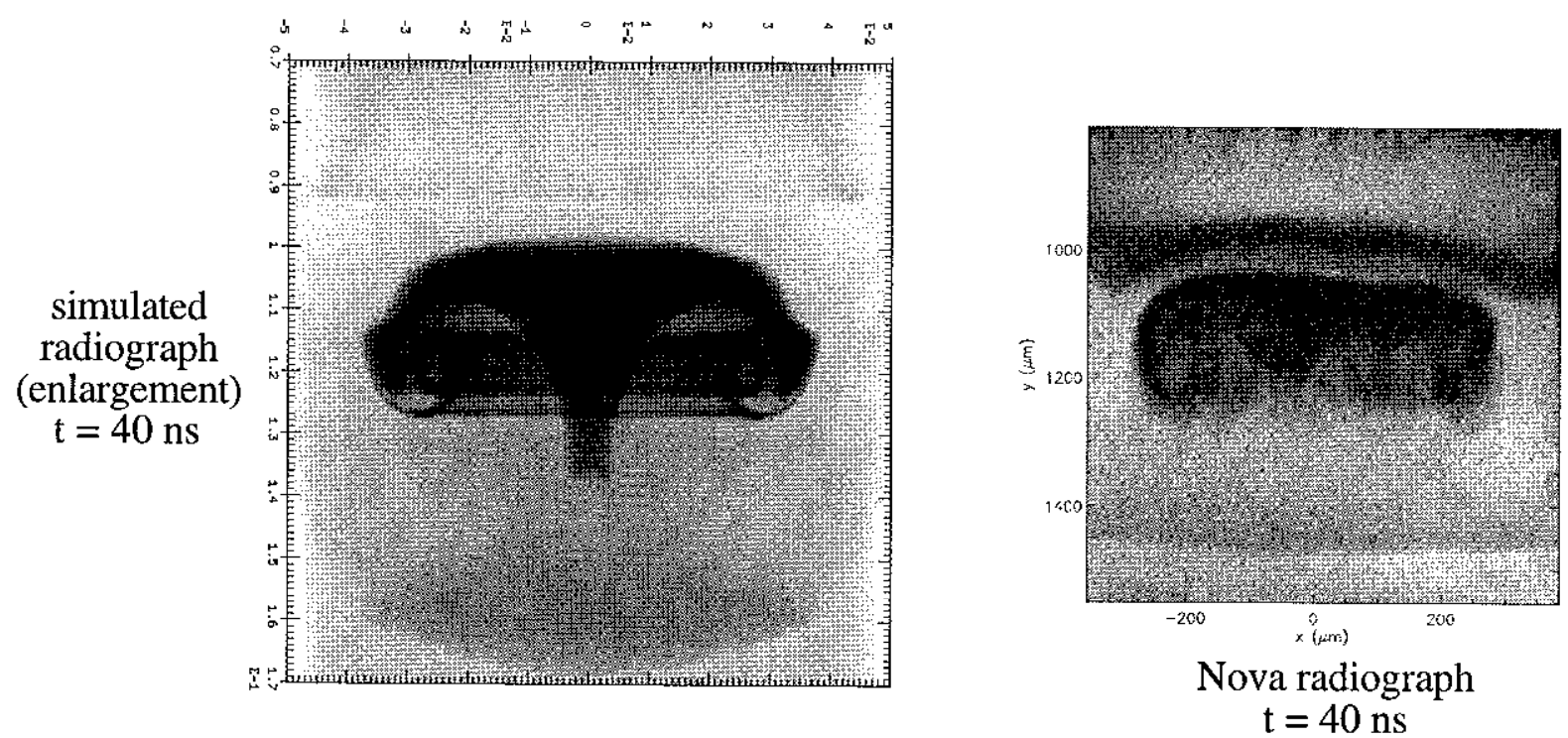

Figure 10. The $400 \mu \mathrm{m}$ diameter $\mathrm{x} 200 \mu \mathrm{m}$ long right-cylinder perturbation design employing drive type B. Simulated and experimental radiographs are shown at $t=25 \mathrm{~ns}$ and $t=40 \mathrm{~ns}$. 

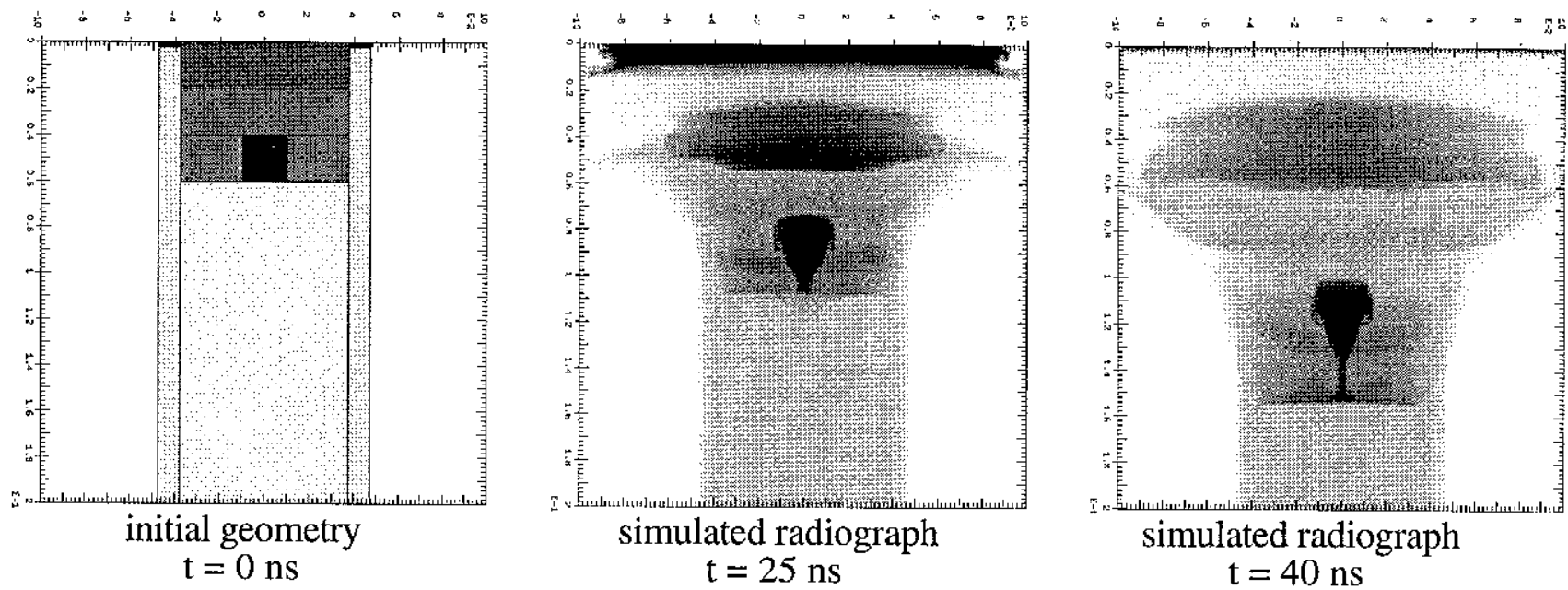

Figure 11. Clear-plastic design in which the right-cylinder perturbation obstruction spans the inside diameter of the shock tube. The brominated-plastic core material appears dark in the radiographs whereas the outer plain-plastic material appears transparent.
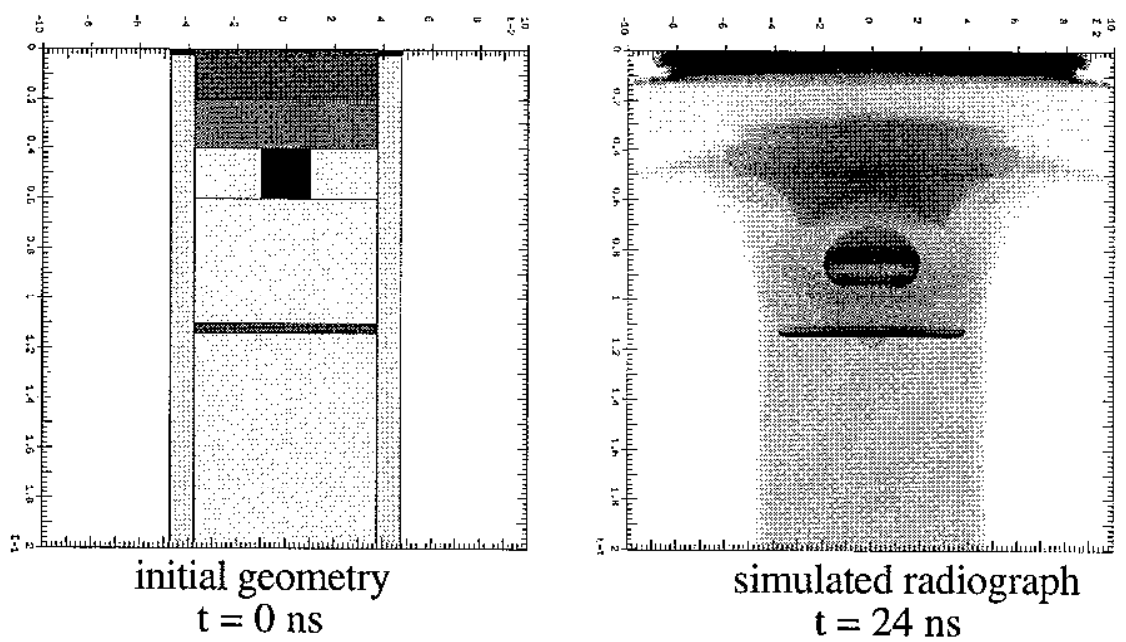

Figure 12. Slab tracer-layer design. A $50 \mu \mathrm{m}$-thick layer of brominated plastic serves as a tracer material to indicate the size of the vortical rollup. 

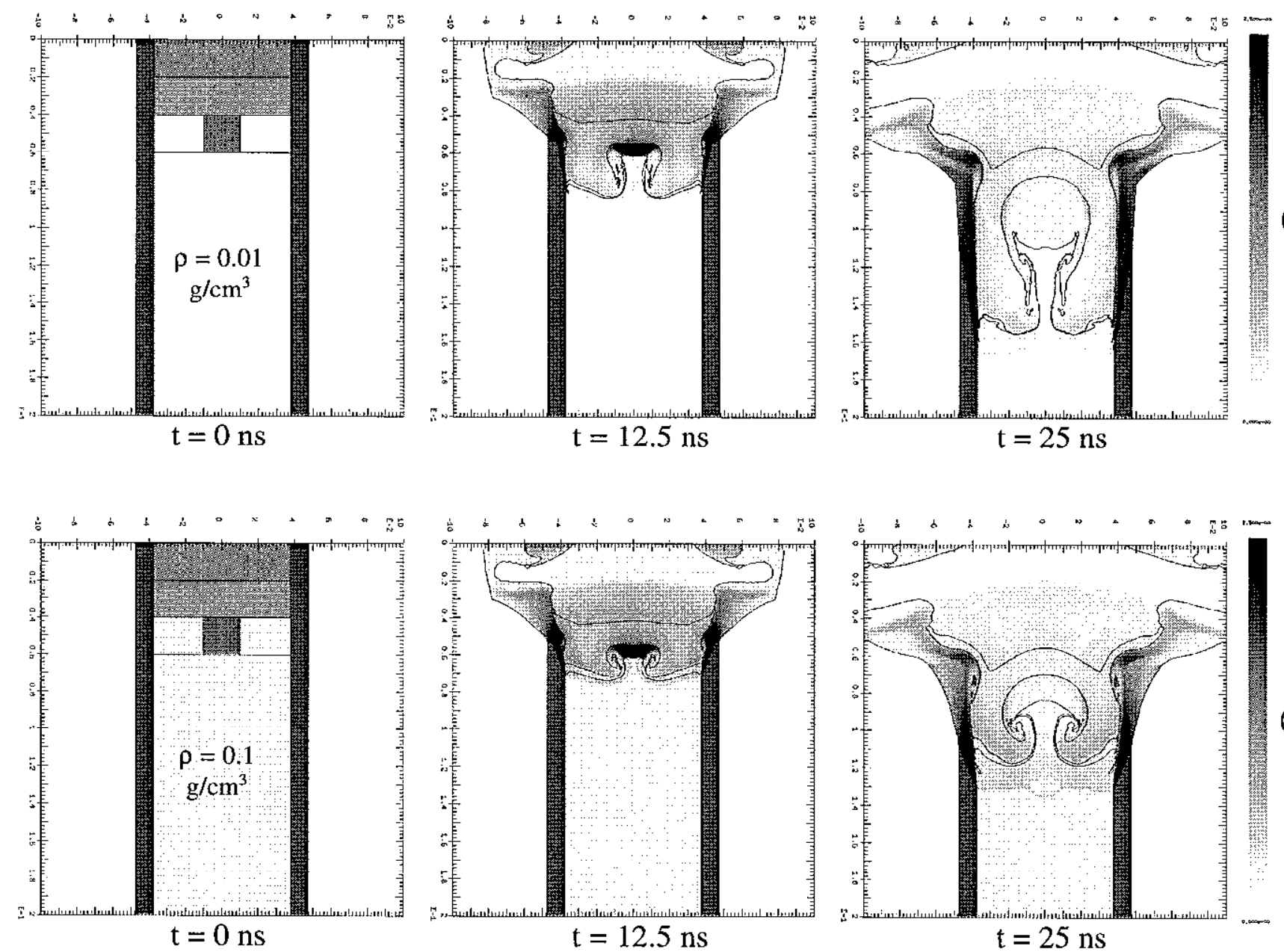

(b)
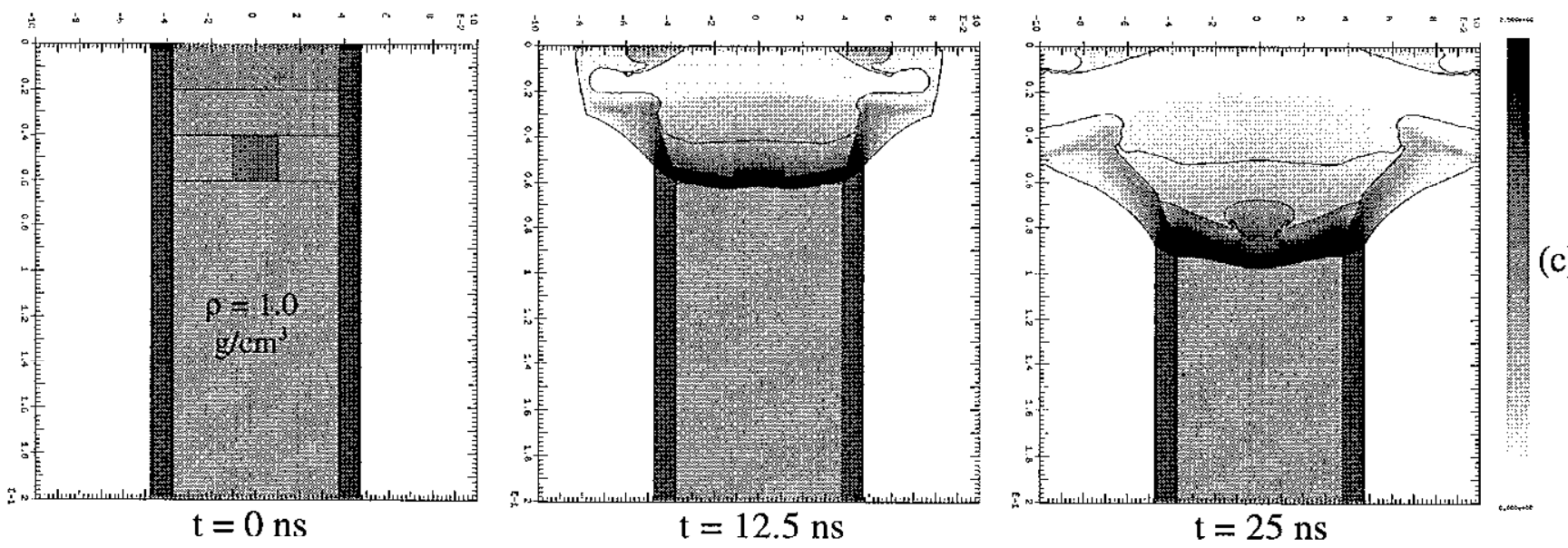

Figure 13. Simulations in which the nominal right-cylinder perturbation design was modified by changing the carbon foam density. Carbon foam densities of (a) $\rho=0.01 \mathrm{~g} / \mathrm{cm}^{3}$, (b) $\rho=0.1 \mathrm{~g} / \mathrm{cm}^{3}$, and (c) $\rho=1.0 \mathrm{~g} / \mathrm{cm}^{3}$ were used. Density contours for the three configurations are shown at $t=0$, 12.5 , and $25 \mathrm{~ns}$. 